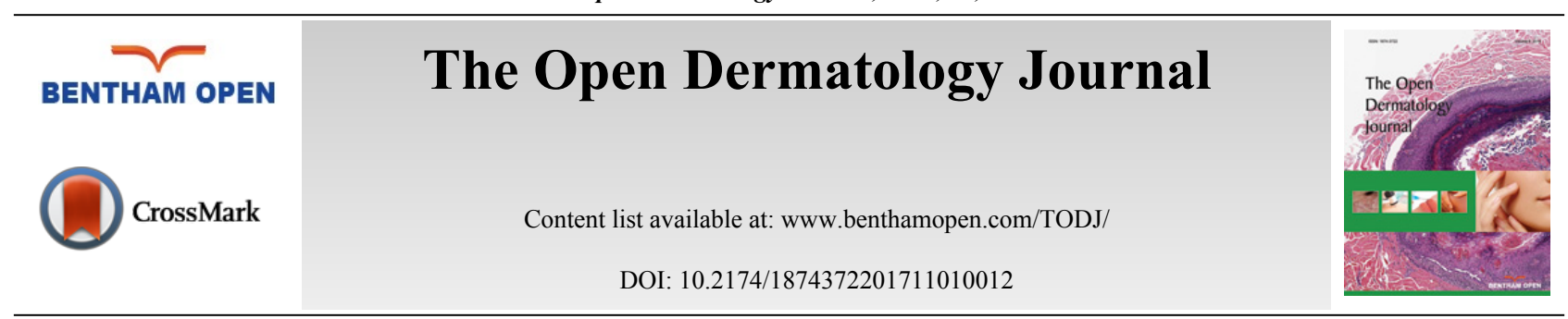

REVIEW ARTICLE

\title{
Assessment of Dietary Supplementation in the Treatment of Vitiligo
}

\author{
Mallory K. Smith ${ }^{1}$, Tasneem F. Mohammad ${ }^{2}$ and Iltefat H. Hamzavi, ${ }^{2, *}$ \\ ${ }^{I}$ Wayne State University School of Medicine, Detroit, MI, USA \\ ${ }^{2}$ Department of Dermatology, Henry Ford Hospital, Detroit, MI, USA
}

Received: December 14, 2016

Revised: February 01, 2017

Accepted: February 03, 2017

\begin{abstract}
:
Background:

Vitiligo is the most common acquired pigmentary disorder in the world. Due to alterations in physical appearance, vitiligo is a psychologically devastating disease. Although treatment options exist, a cure for this disease has yet to be discovered. Of recent interest in vitiligo is the relationship between diet and disease.
\end{abstract}

\section{Objective:}

To review various dietary modifications and supplementation used in the management of vitiligo.

\section{Materials and Methods:}

A thorough evaluation of recent literature using the keywords "vitiligo, diet, supplement, antioxidant, vitamin, mineral, zinc, copper, gluten-free, celiac disease, alternative medicine" in the NCBI PubMed search function was performed.

\section{Results:}

A total of 39 relevant articles were reviewed and critically evaluated.

\section{Conclusion:}

Initial studies regarding the treatment of vitiligo through dietary modification are promising, although further studies are needed in multiple populations to explore the therapeutic value of these interventions.

Keywords: Diet, Vitiligo, Supplementation, Antioxidant, Repigmentation, Management.

\section{INTRODUCTION}

Vitiligo is the most common acquired pigmentary disorder, with an estimated prevalence of $1 \%$ worldwide [1]. The disease is characterized by the development of depigmented macules and patches due to the loss of functioning melanocytes [2,3]. Vitiligo is classified into two broad categories, segmental and non-segmental, with the latter being more common. Segmental vitiligo lesions are generally stable, unilateral, and present in a localized or dermatomal distribution. Non-segmental vitiligo is classically characterized by a waxing and waning course with depigmentation in a symmetric, bilateral pattern, and includes the generalized, acrofacial, and universal variants [4]. The pathogenesis of the disease has yet to be fully elucidated, although an impaired response to oxidative stress, autoimmunity, inflammatory, and neurogenic components may all play a role $[2,4]$. The association between vitiligo and other autoimmune diseases, such as thyroid disease, psoriasis, and inflammatory bowel disease has been established, supporting an autoimmune etiology [5]. Other studies have proposed the relationship between free radicals and an

\footnotetext{
* Address correspondence to this author at the Department of Dermatology, Henry Ford Health System, 3031 West Grand Boulevard, Suite 800, Detroit, MI 48202, USA; Tel: (313) 916-6964; Email: ihamzav1@hfhs.org
} 
impaired response of melanocytes to oxidative stress in the pathogenesis of vitiligo $[2,6]$.

Although there is no cure, a variety of treatment options exist for patients with vitiligo, each with different mechanisms of repigmentation and varying success. These include topical medications such as corticosteroids or immunomodulators, phototherapy, oral medications, autologous melanocyte or epidermal transplant, surgery, and depigmentation [7]. While these therapies are important components in the treatment of vitiligo, the role of dietary modification and supplementation is often overlooked [1,8]. Recent studies have shown that alterations in dietary intake and oral supplementation can be beneficial in the treatment of many diseases, including vitiligo. This article aims to review the various types of dietary modifications and supplementation used in the management of vitiligo.

\section{MATERIALS AND METHODS}

The literature review process was carried out using the keywords "vitiligo, diet, supplement, antioxidant, vitamin, mineral, zinc, copper, gluten free, celiac disease, alternative medicine" in the NCBI PubMed search function. This review utilized articles published within the past ten to fifteen years to obtain recent information and developments. Each article was critically appraised to determine applicability to the topic at hand.

\section{RESULTS}

A total of 44 recent, relevant publications were identified within the scope of this review. Additionally, recent textbooks, as well as USDA and U.S. Department of Health and Human Services online resources were used for reference in the formulation of the discussion and conclusions sections of this article.

\section{DISCUSSION}

\section{A. Antioxidants}

There is evidence to support an imbalance between oxidants and antioxidants in individuals affected by vitiligo. It has been proposed that an overproduction of reactive oxygen species (ROS), in combination with the inherent sensitivity of melanocytes to oxidative stress, may be a mechanism for melanocyte damage and death in vitiligo [9]. Yildirim et al. showed that activity levels of oxidative stress markers such as superoxide dismutase (SOD), glutathione peroxidase (GPx), and malondialdehyde (MDA) were significantly elevated in tissue samples of vitiligo patients [10]. SOD and GPx are enzymes involved in the breakdown of ROS, whereas MDA is produced via lipid peroxidation, often caused by the accumulation of ROS. Other studies observed significantly elevated serum levels of MDA and significantly lower serum levels of SOD, GPx, Vitamins C and E, and overall antioxidant activity in patients with this disease. Serum antioxidant levels were likely lower due to their use in quenching free radicals, whereas MDA was elevated due to the presence of ROS [11].

Imbalances in ox-redox status in vitiligo led to the use of antioxidant supplementation as adjuvant therapy. Oral supplementation with an antioxidant blend of Phyllanthus emblica fruit extracts, vitamin E, and carotenoids, combined with standard topical treatments and/or narrowband ultraviolet B (NBUVB) phototherapy, showed statistically significant increases in repigmentation compared to topical treatment and/or NBUVB alone. Lower levels of serum inflammatory markers were detected in the antioxidant treatment group as well [12].

Alpha-lipoic acid, an over the counter supplement, has also been shown to be beneficial as an adjuvant supplement. This substance acts as a free radical scavenger, lipoxygenase inhibitor, glutathione synthesis promoter, and a factor in the recycling of other antioxidants, such as vitamins $C$ and E [13]. Similarly to the study above, a combination of vitamins $\mathrm{C}$ and $\mathrm{E}$, alpha-lipoic acid, and polyunsaturated fats was used as antioxidant supplementation along with NBUVB phototherapy. A statistically significant increase in repigmentation and decrease in serum reactive oxygen species (ROS) was noted compared to NBUVB phototherapy alone [13].

Supplementation with vitamin E, an antioxidant known to prevent lipid peroxidation, has also been used as an adjunct to NBUVB phototherapy. Study results from Elgoweini et al. reported $72.7 \%$ repigmentation in the vitamin ENBUVB group $(n=12)$, compared to $55.6 \%$ repigmentation in the NBUVB phototherapy group ( $n=12)$. In particular, the average number of treatments required to achieve $50 \%$ repigmentation was significantly lower in the vitamin $\mathrm{E}$ supplementation group than the control group, indicating a more rapid repigmentation with the supplement [14]. Additionally, a significantly lower level of serum MDA was observed in the vitamin E group at the end of the study compared to the beginning, further supporting the value of antioxidant supplementation with traditional phototherapy treatment regimens for vitiligo [14]. Common foods high in vitamin E include sunflower seeds, almonds, hazelnuts, 
peanuts, oils from the aforementioned plants, and breakfast cereals [15]. Caution should be observed in using and recommending this supplement, as vitamin $\mathrm{E}$ has antiplatelet properties and should be used with care in patients with bleeding diathesis or recent surgical procedures. Additionally, vitamin $\mathrm{E}$ is lipid soluble, and overdose is a concern due to the complex metabolism and storage in the body. The recommended daily allowance and upper intake limit for vitamin $\mathrm{E}$ is found in Table $\mathbf{5}$ below.

Polypodium leucotomos extract (PLE) is another antioxidant used to treat dermatologic diseases. Derived from a fern native to Central and South America, this over the counter supplement has been used in the management of conditions including photodermatoses, vitiligo, pigmentary disorders, and as a photoprotectant [16]. PLE contains numerous phenolic compounds with potent antioxidant, anti-inflammatory, and photoprotective properties [17 - 19]. The combination of NBUVB and oral PLE provided statistically significant increases in repigmentation in 50 patients with generalized vitiligo, compared to placebo with NBUVB phototherapy alone. The study concluded that $44 \%$ of the PLE treated patients experienced repigmentation, compared to $27 \%$ in the placebo group. In addition, decreased levels of IL-2, IFN- $\gamma$, and TNF- $\alpha$ were noted in the PLE group, indicating a downregulating effect on cell-mediated immunity [19]. This highlights the interplay between oxidative stress and autoimmunity in the pathogenesis of vitiligo, as well as the utility of oral PLE in disease management. Other studies have shown efficacy in repigmentation with PLE supplementation as well. Pacifico et al. supplemented NBUVB therapy with $480 \mathrm{mg}$ oral P. leucotomos daily for 6 months, and reported increased repigmentation in the PLE group compared to phototherapy alone [20]. In addition, Reyes et al. studied the use of PLE with Psoralen and ultraviolet A (PUVA) treatment, and found that skin repigmentation was significantly higher in the PLE + PUVA group compared to the placebo + PUVA group [21]. Of note, a study by Nestor et al. evaluated the safety of PLE in twenty patients taking $240 \mathrm{mg}$ of PLE daily, and reported an excellent safety profile for this dose range. Gastrointestinal discomfort and pruritus were the most commonly noted side effects [22].

Ginkgo biloba, a supplement available over the counter, is another antioxidant used in the treatment of vitiligo. Parsad et al. studied the efficacy of G. biloba oral supplementation in patients with slowly developing vitiligo lesions. The treatment group took $40 \mathrm{mg}$ of $G$. biloba three times daily for a period of six months. The results indicated that $G$. biloba supplements significantly arrested disease progression compared to placebo, and increased repigmentation with few side effects [23]. A more recent open-label pilot study by Szczurko et al. showed significant improvement in Vitiligo Scoring Index (VASI) scores with $60 \mathrm{mg}$ of G. biloba twice daily for 12 weeks in a total of 12 patients [24]. Additional studies are needed on a larger scale to replicate these findings and provide a better understanding of the mechanism and application of G. biloba in the treatment of vitiligo, although initial findings are promising. Of note to patients and physicians, serious side effects include intracranial hemorrhage and bleeding diathesis, due to the substance's antiplatelet effect. G. biloba has also been known to interfere pharmacologically with anticoagulation medications. Clinical judgment and care should be used in the initiation of this treatment option [25].

For pediatric patients and those who do not desire supplementation in the form of a capsule, increased intake of foods with high antioxidant content is reasonable. Top dietary sources of antioxidants, modified by Wu et al. and reported by Hamzavi et al. are shown below (Table 1) [26, 27]. Although there is limited data correlating antioxidant consumption with health outcomes, these antioxidant-rich foods may be beneficial adjuncts in the treatment of vitiligo.

Table 1. Top 30 foods ranked according to total antioxidant capacities and serving measurements in vitro (C, cooked; g, gram; R, raw. TAC, total antioxidant capacity; umol of TE, micromoles of Trolox equivalents; umol of TE/g, micromoles of Trolox equivalents per gram) [26].

\begin{tabular}{|c|c|c|c|c|}
\hline Rank & Food name & $\begin{array}{c}\text { TAC } \\
\mathbf{u m o l} \\
\text { of } \\
\text { TE/g) }\end{array}$ & Serving size (g) & TAC/serving (umol of TE) \\
\hline 1 & Small red bean & 149.21 & Half cup (92) & 13,727 \\
\hline 2 & Wild blueberry (lowbush) & 92.60 & One cup (145) & 13,427 \\
\hline 3 & Red kidney bean & 144.13 & Half cup (92) & 13,259 \\
\hline 4 & Pinto bean & 123.59 & Half cup (96) & 11,864 \\
\hline 5 & Cultivated blueberry & 62.20 & One cup (145) & 8019 \\
\hline 6 & Cranberry & 94.56 & One cup whole (95) & 7983 \\
\hline 7 & Artichoke (C) & 94.09 & One cup (84) & 7701 \\
\hline 8 & Blackberry & 53.48 & One cup (144) & \\
\hline
\end{tabular}




\begin{tabular}{|c|c|c|c|c|}
\hline Rank & Food name & \begin{tabular}{|c|} 
TAC \\
(umol \\
of \\
TE/g)
\end{tabular} & Serving size (g) & TAC/serving (umol of TE) \\
\hline 9 & Prune & 85.78 & Half cup (85) & 7291 \\
\hline 10 & Raspberry & 49.25 & One cup (123) & 6058 \\
\hline 11 & Strawberry & 35.77 & One cup (166) & 5938 \\
\hline 12 & Red Delicious and Granny Smith apple & 42.75 & One fruit (138) & 5900 \\
\hline 13 & Pecan & 179.40 & One oz (28.4) & 5095 \\
\hline 14 & Russet potato & 13.23 & One potato $(369)$ & 4882 \\
\hline 15 & Sweet cherry & 33.61 & One cup (145) & 4873 \\
\hline 16 & Plum (black) & 73.39 & One fruit (66) & 4844 \\
\hline 17 & Black bean & 80.40 & Half cup (52) & 4181 \\
\hline 18 & Gala apple & 28.28 & One fruit (138) & 3903 \\
\hline 19 & Walnut & 135.41 & One oz (28.4) & 3846 \\
\hline 20 & Golden delicious and Fuji apples & 26.70 & One fruit (138) & 3685 \\
\hline 21 & Deglet Noor dates & 38.95 & Half cup (89) & 3467 \\
\hline 22 & Avocado (Haas) & 19.33 & One fruit (173) & 3344 \\
\hline 23 & Pear, Green and Red Anjou cultivars & 19.11 & One fruit (166) & 3172 \\
\hline 24 & Hazelnut & 96.45 & One oz (28.4) & 2739 \\
\hline 25 & Raab broccoli (R) & 30.84 & Fifth bunch $(85)$ & 2621 \\
\hline 26 & Navel orange & 18.14 & One fruit (140) & 2540 \\
\hline 27 & Fig & 33.83 & Half cup (5) & 2537 \\
\hline 28 & Raisin & 30.37 & Half cup (82) & 2490 \\
\hline 29 & Red cabbage (C) & 31.46 & Half cup (75) & 2359 \\
\hline 30 & Red potato & 10.98 & One potato (213) & 2339 \\
\hline
\end{tabular}

With permission from Hamzavi et al. (2016).

\section{B. Gluten-free Diet}

Autoimmune diseases often co-exist with vitiligo, as reported by Gill et al. [5]. A relationship between vitiligo and celiac disease has been proposed, although evidence has been controversial and incomplete.

Seyhan et al. analyzed children and adolescents with celiac disease, reporting that $9.1 \%$ of the patients were also diagnosed with vitiligo [28]. Following this evidence, Seyhan et al. observed that in a group of 61 patients with vitiligo ( 40 adults and 21 children), there was an $18 \%$ prevalence of concomitant celiac disease seropositivity, with a positivity of $23 \%$ in children and $15 \%$ in adults. However, the relationship was not statistically significant [29]. Additional studies of vitiligo and celiac disease have shown a statistically significant relationship between celiac disease autoantibodies in patients with vitiligo, although further evaluation is needed as this topic is highly controversial at this time [30].

Case reports have provided interesting, although limited, information regarding repigmentation of patients with vitiligo upon the initiation of a gluten-free diet. In one case report, a 9-year old child with celiac disease experienced extensive repigmentation over three years following the initiation of a gluten-free diet. Seven years later, with the continuation of the gluten-free diet, the repigmentation was maintained [31]. Another case report indicated that a 22year old female with acrofacial vitiligo experienced significant repigmentation following the initiation of a gluten-free diet, even though she did not have celiac disease. After numerous topical medications and phototherapy failed to induce repigmentation, the elimination of gluten from her diet, in combination with previously initiated oral dapsone, led to rapid repigmentation, the majority of which occurred in the first month [32].

To manage patients with concomitant celiac disease and vitiligo, a gluten-free diet is recommended for the treatment of the celiac disease, and potential repigmentation of vitiligo. In cases of vitiligo where other treatment options have been exhausted, it may be reasonable to initiate a gluten-free diet.

\section{Minerals: Zinc and Copper}

The role of supplementation with minerals, such as $\mathrm{Zinc}(\mathrm{Zn})$ and Copper $(\mathrm{Cu})$, is another area of interest in vitiligo, although results are controversial. Zinc is a trace element required for homeostasis, with a variety of roles in growth and development, immunomodulation, wound healing, behavior, and taste. It also plays a role in the protection against free radial damage and oxidant-antioxidant balance, as $\mathrm{Zn}$ is a required cofactor for SOD [33]. Copper is also an important 
mineral in homeostasis, with a similarly broad listing of roles in the body. Copper is also considered an antioxidant, as it acts as a coenzyme with antioxidant properties. Both $\mathrm{Zn}$ and $\mathrm{Cu}$ may also be involved in melanogenesis through the

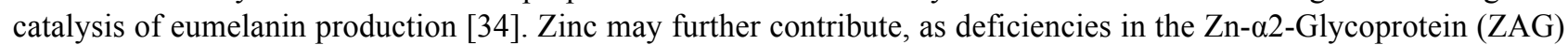
have been reported in people with vitiligo. ZAG is involved in melanocyte proliferation and maturation, with decreased ZAG levels leading to melanocyte detachment. Additionally, treatment with topical corticosteroids, which causes repigmentation, has the ability to increase ZAG activity. Finally, ZAG has been linked to chromosome 7, which contains mutations in certain patients with generalized vitiligo $[35,36]$. This theory has provided an additional basis for the study of $\mathrm{Zn}$ supplementation in patients with vitiligo.

Previous studies, such as that by Shameer et al., evaluated serum Zn levels in vitiligo patients, and found a significant relationship between low $\mathrm{Zn}$ levels and patients with the disease compared to controls [33]. A meta-analysis of Chinese vitiligo patients by Zeng et al. further explored the relationship between both $\mathrm{Zn}$ and Cu levels in this population of patients. Serum $\mathrm{Cu}$ and $\mathrm{Zn}$ were compared between the control and vitiligo patients, with vitiligo patients having significantly lower serum levels of both $\mathrm{Cu}$ and $\mathrm{Zn}$ compared to controls [34]. Although both of these studies indicate some relationship between serum $\mathrm{Zn}$ levels and vitiligo, other findings have determined no significant difference between $\mathrm{Zn}$ levels in vitiligo patients compared to controls, leaving this topic controversial [37].

With respect to treatment using oral Zn, Yaghoobi et al. studied a group of 86 patients with vitiligo, half of whom were treated with topical corticosteroid alone, while the other half was treated with topical corticosteroid plus oral zinc sulfate. A greater improvement in repigmentation was observed in the treatment group, although it was not statistically significant. Overall, this work provided evidence that the combination of topical corticosteroid and $\mathrm{Zn}$ sulfate supplementation was not superior to topical corticosteroid alone, although a positive correlation was observed [38]. Future studies should be continued with larger sample sizes to further understand this potential relationship, in addition to looking at the role $\mathrm{Cu}$ supplementation may play in the treatment of vitiligo. The recommended daily allowance and upper intake limits for $\mathrm{Cu}$ and $\mathrm{Zn}$ are found in Table 5, as well as foods rich in $\mathrm{Cu}$ and $\mathrm{Zn}$ in Tables $\mathbf{2}$ and $\mathbf{3}$.

Table 2. Selected foods rich in Copper [15].

\begin{tabular}{|c|c|c|c|c|}
\hline Foods & Measure & $\begin{array}{c}\text { Copper, } \mathrm{Cu}(\mathrm{mg}) \text { per } \\
\text { measure }\end{array}$ & $\%$ RDA (0.9 mg/day) & $\begin{array}{c}\% \text { UL (10 } \\
\text { mg/day) }\end{array}$ \\
\hline Beef variety meats and by-products, cooked & 1.0 slice & 11.816 & 1312 & 118 \\
\hline Lamb liver, cooked & $3.0 \mathrm{oz}$ & 11.390 & 1265 & 114 \\
\hline Lamb variety meats and by-products, cooked & $3.0 \mathrm{oz}$ & 8.356 & 928 & 84 \\
\hline Seaweed, spirulina & 1.0 cup & 6.832 & 759 & 68 \\
\hline Mollusks, oyster, cooked & $3.0 \mathrm{oz}$ & 4.851 & 539 & 49 \\
\hline Beef sweetbread, cooked & $3.0 \mathrm{oz}$ & 4.335 & 481 & 43 \\
\hline Cocoa, dry powder, unsweetened & 1 cup & 3.258 & 362 & 33 \\
\hline Cashew nuts, dry roasted & 1.0 cup, halves and whole & 3.041 & 338 & 30 \\
\hline Sunflower seed kernels, toasted & 1.0 cup & 2.458 & 273 & 25 \\
\hline Buckwheat & 1.0 cup & 1.870 & 207 & 19 \\
\hline
\end{tabular}

Table 3. Selected foods rich in Zinc [15].

\begin{tabular}{|c|c|c|c|c|}
\hline Foods & Measure & Zinc, Zn (mg) per measure & \% RDA (8 mg/day) & \% UL (10 mg/day) \\
\hline Mollusks, oyster, eastern, canned & $3.0 \mathrm{oz}$ & 77.31 & 966 & 773 \\
\hline Beef, bottom sirloin & 1.0 roast (690g raw meat) & 26.57 & 332 & 267 \\
\hline Cereals (ready-to-eat) & $0.75-1.0 \mathrm{cup}$ & 15.0 & 188 & 150 \\
\hline Wheat germ & $1.0 \mathrm{cup}$ & 14.13 & 177 & 141 \\
\hline Turkey breast, cooked & $1.0 \mathrm{breast}$ & 13.12 & 164 & 131 \\
\hline Sesame seed kernels, toasted & $1.0 \mathrm{cup}$ & 13.09 & 142 & 131 \\
\hline Lamb, foreshank, cooked & 1.0 piece, cooked & 11.38 & 133 & 114 \\
\hline Ground Turkey & $1.0 \mathrm{lb}$ & 10.66 & 127 & 107 \\
\hline Crustaceans, crab, cooked & $1.0 \mathrm{leg}$ & 10.21 & 118 & 102 \\
\hline Peanuts, oil roasted & $1.0 \mathrm{cup}$ & 9.47 & & 95 \\
\hline
\end{tabular}

\section{Vitamin D}

Vitamin D plays a variety of roles in the human body, including calcium and bone homeostasis, cell proliferation 
and growth, and melanogenesis. It is theorized that vitamin D plays an active role in melanogenesis, as this hormone is synthesized within the skin after exposure to UVB light, and perhaps involved in the maturation of melanocytes to produce melanin and undergo appropriate differentiation [39]. Vitamin D is also thought to play a role in immunomodulation, although the mechanism is not well understood. Studies have indicated the use of vitamin D in the treatment of autoimmune diseases such as systemic lupus erythematosus, diabetes mellitus, rheumatoid arthritis, and multiple sclerosis, although this topic is highly controversial [40].

In particular, Silverberg et al. studied the relationship between serum 25-hydroxyvitamin D levels and the presence of autoimmune diseases. It was found that very low 25 -hydroxyvitamin D level $(<15 \mathrm{ng} / \mathrm{mL})$ in patients with vitiligo was associated with comorbid autoimmune disease, such as thyroid disease, Sjögren syndrome, rheumatoid arthritis, alopecia areata, and inflammatory bowel disease [41].

Looking at serum levels of vitamin D in vitiligo from a different perspective, Sehrawat et al. analyzed the levels of 25-hydroxyvitamin D in Indian patients with vitiligo throughout a 12-week phototherapy treatment consisting of three NBUVB sessions per week. Interestingly, all of the patients had sub-normal levels of serum 25-hydroxyvitamin D at the start of the study, but throughout the course of NBUVB treatment, serum 25-hydroxyvitamin D levels increased. Additionally, this group reported a significant improvement in VASI scores as repigmentation occurred throughout the treatment. Although the increasing trend of serum vitamin D levels was insignificant, this study provided interesting insight into the potential role of vitamin $\mathrm{D}$ in the repigmentation of vitiligo lesions, perhaps through the regulation of photo-induced melanogenesis [42].

Additional studies by Finamor et al. supported this theory, as they assessed the effect of long-term administration of 25-hydroxyvitamin D in the treatment of patients with vitiligo and psoriasis. Sixteen vitiligo patients were treated with 35,000 IU of 25-hydroxyvitamin D daily for six months, and as expected, measured serum levels of vitamin D increased significantly throughout the study. Additionally, eleven out of the sixteen patients with vitiligo experienced $25-75 \%$ repigmentation during this time period [43]. Increases in serum vitamin D levels were associated with corresponding repigmentation. This study raises the possibility of vitamin D supplementation as a treatment option for patients with vitiligo [43]. However, further studies are needed for a more complete understanding of the mechanism and clinical utility of vitamin D supplementation, as this topic is controversial and in need of further evidence. Of note, vitamin D is a fat-soluble vitamin, which can reach toxic levels if ingested in excess. Toxicity is defined as vitamin D levels higher than $150 \mathrm{ng} / \mathrm{mL}$, or the presence of associated symptoms such as hypercalcemia, nausea, constipation, or weakness. Although daily recommendations are controversial, toxicity is unlikely in an adult when taking doses less than 10,000 IU daily. Additionally, a safe daily limit, as recommended by the Institute of Medicine, is 4,000 IU daily for individuals older than 8 years of age $[44,45]$. Table $\mathbf{4}$ and $\mathbf{5}$ lists the recommended daily allowance for vitamin D as well as foods rich in vitamin D.

Table 4. Selected foods rich in Vitamin D [15].

\begin{tabular}{|c|c|c|c|c|}
\hline Foods & Measure & $\begin{array}{c}\text { Vitamin D (D2 + D3) (ug) per } \\
\text { measure }\end{array}$ & $\begin{array}{c}\text { \% RDA (600 IU or 15 } \\
\text { ug) }\end{array}$ & \% UL (40,000 ug/day) \\
\hline Mushrooms - brown, Italian or crimini (raw) & $1.0 \mathrm{cup}$ & 27.8 & 185 & 0.70 \\
\hline Salmon, canned & $3.0 \mathrm{oz}$ & 18.3 & 122 & 0.46 \\
\hline Smoked whitefish & $1.0 \mathrm{cup}$ & 17.4 & 116 & 0.43 \\
\hline Mushrooms - portabella, grilled & 1.0 cup sliced & 15.9 & 106 & 0.40 \\
\hline Salmon, cooked & $3.0 \mathrm{oz}$ & 14.1 & 94 & 0.35 \\
\hline Swordfish, cooked & $3.0 \mathrm{oz}$ & 14.1 & 94 & 0.35 \\
\hline Rainbow trout, cooked & 1.0 fillet & 13.5 & 90 & 0.34 \\
\hline Tuna, canned & $1.0 \mathrm{cup}$ & 9.8 & 65 & 0.25 \\
\hline Milk (whole), added vitamin D & $0.25 \mathrm{cup}$ & 3.4 & 23 & 0.23 \\
\hline Hardboiled egg & $1.0 \mathrm{cup}$ & 3.0 & 20 & 0.07 \\
\hline
\end{tabular}

\section{E. Alternative Medicine}

A variety of alternative treatment practices have been used as therapeutic remedies for vitiligo, although there is a paucity of clinical studies in the literature. Many of these treatments utilize herbs and extracts with photosensitizing properties in conjunction with natural sunlight to induce repigmentation. These practices include Ayurvedic, Unani, and Traditional Chinese medicine (TCM) techniques [26]. 
Ayurvedic medicine was developed in India thousands of years ago and is still practiced today. This form of medicine takes a holistic approach toward the patient, incorporating spirit, body, mind, and emotions and often involves dietary modifications. Dietotherapy for vitiligo includes Saman Chikitsa, which consists of two parts, Kastha Aushadhi and Saman Chikitsa. Kastha Aushadhi uses herbal supplements as photosensitizing and blood-purifying treatments, whereas Rasa Shastra is a method of formulating combinations of herbs and minerals for immunosuppression. The list of herbs used in both is extensive, and the dosing and regimens are often Ayurvedic practitioner and pharmacy dependent, making it difficult to provide exact regimens. An additional form of Ayurvedic treatment for vitiligo is Sodhan Chikitsa, a method of purification of the body through the use of vomiting, purgation, enemas, and bloodletting. This practice involves the use of laxatives, bran, flaxseed, fruits, and many other dietary agents [26].

Unani medicine is derived from influences of Greek, Islamic, and Arabic cultures, and is practiced today in many parts of the Middle East, South Africa and regions of Asia. According to this practice, the human body is composed of the following four components: air, water, fire, and earth. An imbalance of these elements is thought to be the causative factor in illness and disease. Treatment regimens were developed surrounding this ideology. In addition to Iiaj Nafansi (psychotherapy), Unani medicine recommends the use of Iiaj Bil Ghiza (dietotherapy) for the treatment of vitiligo. Helpful foods according to this practice are listed as follows; warm, easily digestible foods, goat and bird meat, and digestive aids with meals. Additionally, the avoidance of fresh fish, fruits, vegetables, and foods high in fat is recommended, as these foods are theorized to cause an imbalance in the elements of the body [26].

Traditional Chinese Medicine (TCM), which was developed and practiced in China, views the human body as a combination of two opposing but complementary forces, the Ying and Yang. An imbalance in the Ying and Yang is thought to produce illness and disease. This practice uses a holistic approach in patient management, by combining physical, oral, and acupuncture practices in the management or prevention of disease. In particular, TCM dietary treatments for vitiligo include the use of herbal supplements, such as Golden Serpent fern extract in combination with NBUVB, Ginkgo biloba, and Xiaobai Mixture, a blend of walnut, flowers, black sesame, black beans, duckweed, Sweetgum fruit, and plums [26]. Chen et al. assessed the effectiveness of a combination of oral TCM with NBUVB therapy compared to NBUVB alone in a systematic review with meta-analysis of randomized controlled trials. This study determined that in regards to repigmentation, CHM combined with phototherapy was superior to phototherapy alone in the treatment of vitiligo $(\mathrm{p}<0.00001)$ [46].

Supplementation regimens in these practices are difficult to quantify and not commonly used in the United States. Future studies are necessary and may further elucidate the role of alternative medicine in the management of vitiligo.

\section{CONCLUSION}

Vitiligo is a complex disease with a multitude of components contributing to its pathogenesis. Alterations in the diet may target many of these components, and recent evidence suggests that dietary modifications and supplementation can be beneficial in the treatment of vitiligo, especially as adjuvant therapies to phototherapy or topical regimens. Although initial findings with these supplements are promising, further studies in multiple populations are needed to explore the therapeutic value of these interventions and further develop these options to optimize the management of this psychologically devastating disease.

Table 5. Compiled list of Recommended Dietary Allowances (RDA) and Upper Intake Level (UL) of discussed vitamins and minerals [47].

\begin{tabular}{|c|c|c|}
\hline Substance & RDA: 19-30 years, 30-50 years, and 50-70 years & UL: 19-30 years, 30-50 years, and 50-70 years \\
\hline Copper & $900 \mathrm{ug} /$ day & $10,000 \mathrm{ug} / \mathrm{day}$ \\
\hline Vitamin C & Males: $90 \mathrm{mg} /$ day, Females: $75 \mathrm{mg} /$ day & $2,000 \mathrm{mg} /$ day \\
\hline Vitamin D & $600 \mathrm{IU} /$ day & $4,000 \mathrm{IU} / \mathrm{day}$ \\
\hline Vitamin E & $15 \mathrm{mg} /$ day & $1,000 \mathrm{mg} / \mathrm{day}$ \\
\hline Zinc & Males: $11 \mathrm{mg} /$ day, Females: $8 \mathrm{mg} /$ day & $40 \mathrm{mg} /$ day \\
\hline
\end{tabular}

\section{CONFLICT OF INTEREST}

Dr. Hamzavi is an investigator for Estee-Lauder, Ferndale, Johnson and Johnson, and Allergan, and has received equipment from Johnson and Johnson. Dr. Mohammad is a subinvestigator for Estee-Lauder, Ferndale, and Allergan. Ms. Smith has no conflicts of interest to report. 


\section{ACKNOWLEDGEMENTS}

Declared none.

\section{REFERENCES}

[1] Ezzedine K, Eleftheriadou V, Whitton M, van Geel N. Vitiligo. Lancet 2015; 386(9988): 74-84. [http://dx.doi.org/10.1016/S0140-6736(14)60763-7] [PMID: 25596811]

[2] Alikhan A, Felsten LM, Daly M, Petronic-Rosic V. Vitiligo: A comprehensive overview Part I. Introduction, epidemiology, quality of life, diagnosis, differential diagnosis, associations, histopathology, etiology, and work-up. J Am Acad Dermatol 2011; 65(3): 473-91. [http://dx.doi.org/10.1016/j.jaad.2010.11.061] [PMID: 21839315]

[3] Gauthier Y, Cario Andre M, Taïeb A. A critical appraisal of vitiligo etiologic theories. Is melanocyte loss a melanocytorrhagy? Pigment Cell Res 2003; 16(4): 322-32. [http://dx.doi.org/10.1034/j.1600-0749.2003.00070.x] [PMID: 12859615]

[4] Taïeb A, Picardo M. Clinical practice. Vitiligo. N Engl J Med 2009; 360(2): 160-9. [PMID: 19129529]

[5] Gill L, Zarbo A, Isedeh P, Jacobsen G, Lim HW, Hamzavi I. Comorbid autoimmune diseases in patients with vitiligo: A cross-sectional study. J Am Acad Dermatol 2016; 74(2): 295-302. [http://dx.doi.org/10.1016/j.jaad.2015.08.063] [PMID: 26518171]

[6] Güntaş G, Engin B, Ekmekçi OB, et al. Evaluation of advanced oxidation protein products, prooxidant-antioxidant balance, and total antioxidant capacity in untreated vitiligo patients. Ann Dermatol 2015;27(2): 178-83. [http://dx.doi.org/10.5021/ad.2015.27.2.178] [PMID: 25834357]

[7] Sisti A, Sisti G, Oranges CM. Effectiveness and safety of topical tacrolimus monotherapy for repigmentation in vitiligo: A comprehensive literature review. An Bras Dermatol 2016; 91(2): 187-95. [http://dx.doi.org/10.1590/abd1806-4841.20164012] [PMID: 27192518]

[8] Felsten LM, Alikhan A, Petronic-Rosic V. Vitiligo: a comprehensive overview Part II: treatment options and approach to treatment. J Am Acad Dermatol 2011; 65(3): 493-514. [http://dx.doi.org/10.1016/j.jaad.2010.10.043] [PMID: 21839316]

[9] Xie H, Zhou F, Liu L, et al. Vitiligo: How do oxidative stress-induced autoantigens trigger autoimmunity? J Dermatol Sci 2016; 81(1): 3-9. [http://dx.doi.org/10.1016/j.jdermsci.2015.09.003] [PMID: 26387449]

[10] Yildirim M, Baysal V, Inaloz HS, Can M. The role of oxidants and antioxidants in generalized vitiligo at tissue level. J Eur Acad Dermatol Venereol 2004; 18(6): 683-6.

[http://dx.doi.org/10.1111/j.1468-3083.2004.01080.x] [PMID: 15482295]

[11] Khan R, Satyam A, Gupta S, Sharma VK, Sharma A. Circulatory levels of antioxidants and lipid peroxidation in Indian patients with generalized and localized vitiligo. Arch Dermatol Res 2009; 301(10): 731-7. [http://dx.doi.org/10.1007/s00403-009-0964-4] [PMID: 19488773]

[12] Colucci R, Dragoni F, Conti R, Pisaneschi L, Lazzeri L, Moretti S. Evaluation of an oral supplement containing Phyllanthus emblica fruit extracts, vitamin E, and carotenoids in vitiligo treatment. Dermatol Ther (Heidelb) 2015; 28(1): 17-21. [http://dx.doi.org/10.1111/dth.12172] [PMID: 25285994]

[13] DellAnna ML, Mastrofrancesco A, Sala R, et al. Antioxidants and narrow band-UVB in the treatment of vitiligo: a double-blind placebo controlled trial. Clin Exp Dermatol 2007; 32(6): 631-6. [http://dx.doi.org/10.1111/j.1365-2230.2007.02514.x] [PMID: 17953631]

[14] Elgoweini M, Nour El Din N. Response of vitiligo to narrowband ultraviolet B and oral antioxidants. J Clin Pharmacol 2009; 49(7): 852-5. [http://dx.doi.org/10.1177/0091270009335769] [PMID: 19553407]

[15] USDA Food Composition Databases Available from: https://ndb.nal.usda.gov/ndb/nutrients/index 2016 [09/15/2016];

[16] Choudhry SZ, Bhatia N, Ceilley R, et al. Role of oral Polypodium leucotomos extract in dermatologic diseases: A review of the literature. J Drugs Dermatol 2014; 13(2): 148-53. [PMID: 24509964]

[17] Berman B, Ellis C, Elmets C. Polypodium LeucotomosAn Overview of Basic Investigative Findings. J Drugs Dermatol 2016 ; $15(2)$ : $224-8$. [PMID: 26885792]

[18] Nestor M, Bucay V, Callender V, Cohen JL, Sadick N, Waldorf H. Polypodium leucotomos as an Adjunct Treatment of Pigmentary Disorders. J Clin Aesthet Dermatol 2014; 7(3): 13-7. [PMID: 24688621]

[19] Middelkamp-Hup MA, Bos JD, Rius-Diaz F, Gonzalez S, Westerhof W. Treatment of vitiligo vulgaris with narrow-band UVB and oral Polypodium leucotomos extract: a randomized double-blind placebo-controlled study. J Eur Acad Dermatol Venereol 2007; 21(7): $942-50$. [http://dx.doi.org/10.1111/j.1468-3083.2006.02132.x] [PMID: 17659004]

[20] Pacifico AI, Paro Vidolin A, Leone G. Combined treatment of narrowband ultraviolet B light (NBUVB) phototherapy and oral polypodium leucotomos extract versus NB UVB phototherapy alone in the treatment of patients with vitiligo. J Am Acad Dermatol $2009 ; 60$. 
[21] Reyes E, Jaén P, de las Heras E, et al. Systemic immunomodulatory effects of Polypodium leucotomos as an adjuvant to PUVA therapy in generalized vitiligo: A pilot study. J Dermatol Sci 2006; 41(3): 213-6. [http://dx.doi.org/10.1016/j.jdermsci.2005.12.006] [PMID: 16423508]

[22] Nestor MS, Berman B, Swenson N. Safety and efficacy of oral polypodium leucotomos extract in healthy adult subjects. J Clin Aesthet Dermatol 2015; 8(2): 19-23.

[PMID: 25741399]

[23] Parsad D, Pandhi R, Juneja A. Effectiveness of oral Ginkgo biloba in treating limited, slowly spreading vitiligo. Clin Exp Dermatol 2003; 28(3): 285-7.

[http://dx.doi.org/10.1046/j.1365-2230.2003.01207.x] [PMID: 12780716]

[24] Szczurko O, Shear N, Taddio A, Boon H. Ginkgo biloba for the treatment of vitilgo vulgaris: an open label pilot clinical trial. BMC Complement Altern Med 2011; 11: 21. [http://dx.doi.org/10.1186/1472-6882-11-21] [PMID: 21406109]

[25] Ernst E. The risk-benefit profile of commonly used herbal therapies: Ginkgo, St. Johns Wort, Ginseng, Echinacea, Saw Palmetto, and Kava. Ann Intern Med 2002; 136(1): 42-53.

[http://dx.doi.org/10.7326/0003-4819-136-1-200201010-00010] [PMID: 11777363]

[26] Hamzavi IH. BH, Isedeh PN Handbook of Vitiligo. London: JP Medical Ltd. 2016.

[27] Wu X, Beecher GR, Holden JM, Haytowitz DB, Gebhardt SE, Prior RL. Lipophilic and hydrophilic antioxidant capacities of common foods in the United States. J Agric Food Chem 2004; 52(12): 4026-37. [http://dx.doi.org/10.1021/jf049696w] [PMID: 15186133]

[28] Seyhan M, Erdem T, Ertekin V, Selimoğlu MA. The mucocutaneous manifestations associated with celiac disease in childhood and adolescence. Pediatr Dermatol 2007; 24(1): 28-33. [http://dx.doi.org/10.1111/j.1525-1470.2007.00328.x] [PMID: 17300645]

[29] Seyhan M, Kandi B, Akbulut H, Selımoğlu MA, Karincaoğlu M. Is celiac disease common in patients with vitiligo? Turk J Gastroenterol 2011; 22(1): 105-6.

[http://dx.doi.org/10.4318/tjg.2011.0169] [PMID: 21480124]

[30] Shahmoradi Z, Najafian J, Naeini FF, Fahimipour F. Vitiligo and autoantibodies of celiac disease. Int J Prev Med 2013; 4(2): 200-3. [PMID: 23543680]

[31] Rodríguez-García C, González-Hernández S, Pérez-Robayna N, Guimerá F, Fagundo E, Sánchez R. Repigmentation of vitiligo lesions in a child with celiac disease after a gluten-free diet. Pediatr Dermatol 2011; 28(2): 209-10. [http://dx.doi.org/10.1111/j.1525-1470.2011.01388.x] [PMID: 21504457]

[32] Khandalavala BN, Nirmalraj MC. Rapid partial repigmentation of vitiligo in a young female adult with a gluten-free diet. Case Rep Dermatol $2014 ; 6(3): 283-7$ [http://dx.doi.org/10.1159/000370303] [PMID: 25685131]

[33] Shameer P, Prasad PV, Kaviarasan PK. Serum zinc level in vitiligo: a case control study. Indian J Dermatol Venereol Leprol 2005; 71(3): 206-7.

[http://dx.doi.org/10.4103/0378-6323.16243] [PMID: 16394417]

[34] Zeng Q, Yin J, Fan F, et al. Decreased copper and zinc in sera of Chinese vitiligo patients: a meta-analysis. J Dermatol 2014; 41(3): 245-51. [http://dx.doi.org/10.1111/1346-8138.12392] [PMID: 24517587]

[35] Mohammed GF, Gomaa AH, Al-Dhubaibi MS. Highlights in pathogenesis of vitiligo. World J Clin Cases 2015; 3(3): 221-30. [http://dx.doi.org/10.12998/wjcc.v3.i3.221] [PMID: 25789295]

[36] Hassan MI, Waheed A, Yadav S, Singh TP, Ahmad F. Zinc alpha 2-glycoprotein: a multidisciplinary protein. Mol Cancer Res 2008; 6(6): $892-906$.

[http://dx.doi.org/10.1158/1541-7786.MCR-07-2195] [PMID: 18567794]

[37] Arora PN, Dhillon KS, Rajan SR, Sayal SK, Das AL. Serum Zinc Levels in Cutaneous Disorders. Med J Armed Forces India 2002; 58(4): 304-6.

[http://dx.doi.org/10.1016/S0377-1237(02)80083-1] [PMID: 27407419]

[38] Yaghoobi R, Omidian M, Bagherani N. Original article title: Comparison of therapeutic efficacy of topical corticosteroid and oral zinc sulfatetopical corticosteroid combination in the treatment of vitiligo patients: a clinical trial. BMC Dermatol 2011; 11: 7. [http://dx.doi.org/10.1186/1471-5945-11-7] [PMID: 21453467]

[39] Parsad D, Kanwar AJ. Topical vitamin D analogues in the treatment of vitiligo. Pigment Cell Melanoma Res 2009; 22(4): 487-8. [http://dx.doi.org/10.1111/j.1755-148X.2009.00579.x] [PMID: 19422605]

[40] AlGhamdi K, Kumar A, Moussa N. The role of vitamin D in melanogenesis with an emphasis on vitiligo. Indian J Dermatol Venereol Leprol 2013; 79(6): 750-8.

[http://dx.doi.org/10.4103/0378-6323.120720] [PMID: 24177606]

[41] Silverberg JI, Silverberg AI, Malka E, Silverberg NB. A pilot study assessing the role of 25 hydroxy vitamin D levels in patients with vitiligo vulgaris. J Am Acad Dermatol 2010; 62(6): 937-41. [http://dx.doi.org/10.1016/j.jaad.2009.11.024] [PMID: 20466170] 
[42] Sehrawat M, Arora TC, Chauhan A, Kar HK, Poonia A, Jairath V. Correlation of Vitamin D Levels with Pigmentation in Vitiligo Patients Treated with NBUVB Therapy. ISRN Dermatol 2014; 2014: 493213. [http://dx.doi.org/10.1155/2014/493213] [PMID: 25006488]

[43] Finamor DC, Sinigaglia-Coimbra R, Neves LC, et al. A pilot study assessing the effect of prolonged administration of high daily doses of vitamin D on the clinical course of vitiligo and psoriasis. Dermatoendocrinol 2013; 5(1): 222-34. [http://dx.doi.org/10.4161/derm.24808] [PMID: 24494059]

[44] Reddy KK, Gilchrest BA. Iatrogenic effects of photoprotection recommendations on skin cancer development, vitamin D levels, and general health. Clin Dermatol 2011; 29(6): 644-51.

[http://dx.doi.org/10.1016/j.clindermatol.2011.08.027] [PMID: 22014986]

[45] Holick MF. Vitamin D deficiency. N Engl J Med 2007; 357(3): 266-81. [http://dx.doi.org/10.1056/NEJMra070553] [PMID: 17634462]

[46] Chen YJ, Chen YY, Wu CY, Chi CC. Oral Chinese herbal medicine in combination with phototherapy for vitiligo: A systematic review and meta-analysis of randomized controlled trials. Complement Ther Med 2016; 26: 21-7. [http://dx.doi.org/10.1016/j.ctim.2016.02.009] [PMID: 27261977]

[47] Recommendations N. Nutrient Recommendations: Dietary Reference Intakes (DRI): U.S. Department of Health and Human Services; [Available from: https://ods.od.nih.gov/Health_Information/Dietary_Reference_Intakes.aspx 2016.

(C) 2017 Smith et al.

This is an open access article distributed under the terms of the Creative Commons Attribution 4.0 International Public License (CC-BY 4.0), a copy of which is available at: (https://creativecommons.org/licenses/by/4.0/legalcode). This license permits unrestricted use, distribution, and reproduction in any medium, provided the original author and source are credited. 